Bull. Korean Math. Soc. 51 (2014), No. 1, pp. 207-211

http://dx.doi.org/10.4134/BKMS.2014.51.1.207

\title{
IDENTITIES WITH ADDITIVE MAPPINGS IN SEMIPRIME RINGS
}

\author{
AJda Fošner And NADEem uR Rehman
}

\begin{abstract}
The aim of this paper is to prove the next result. Let $n>1$ be an integer and let $R$ be a $n$ !-torsion free semiprime ring. Suppose that $f: R \rightarrow R$ is an additive mapping satisfying the relation $\left[f(x), x^{n}\right]=0$ for all $x \in R$. Then $f$ is commuting on $R$.
\end{abstract}

\section{Introduction and the main theorem}

Throughout, $R$ will represent an associative ring with a center $Z(R)$. Let $n>1$ be an integer. A ring $R$ is $n$-torsion free if $n x=0, x \in R$, implies $x=0$. The Lie product (or a commutator) of elements $x, y \in R$ will be denoted by $[x, y]$ (i.e., $[x, y]=x y-y x$ ). Recall that a ring $R$ is prime if $a R b=\{0\}$, $a, b \in R$, implies that either $a=0$ or $b=0$. Furthermore, a ring $R$ is called semiprime if $a R a=\{0\}, a \in R$, implies $a=0$. We will denote by $C$ and $Q$ the extended centroid and the maximal right ring of quotients of a semiprime ring $R$, respectively. For the explanation of the extended centroid as well as the maximal right ring of quotients of a semiprime ring we refer the reader to [4]. As usual, the socle of a ring $R$ will be denoted by $\operatorname{soc}(R)$.

An additive mapping $D: R \rightarrow R$ is called a derivation on $R$ if $D(x y)=$ $D(x) y+x D(y)$ holds for all pairs $x, y \in R$. An additive mapping $f: R \rightarrow$ $R$ is called centralizing on $R$ if $[f(x), x] \in Z(R)$ holds for all $x \in R$. In a special case, when $[f(x), x]=0$ for all $x \in R$, the mapping $f$ is said to be commuting on $R$. A classical result of Posner [21] (Posner's second theorem) states that the existence of a nonzero centralizing derivation on a prime ring forces the ring to be commutative. Posner's second theorem in general cannot be proved for semiprime rings as shows the following example. Let $R_{1}$ and $R_{2}$ be prime rings with $R_{1}$ commutative and set $R=R_{1} \oplus R_{2}$. Further, let $D_{1}: R_{1} \rightarrow R_{1}$ be a nonzero derivation. A mapping $D: R \rightarrow R$ defined by

Received February 12, 2013; Revised April 25, 2013

2010 Mathematics Subject Classification. 16N60, 16R50.

Key words and phrases. prime ring, semiprime ring, additive mapping, centralizing mapping, commuting mapping.

This Research was supported by bilateral research program between India and Slovenia (Grant No. INT/SLOVENIA/p-18/2009). 
$D\left(\left(r_{1}, r_{2}\right)\right)=\left(D_{1}\left(r_{1}\right), 0\right)$ is then a nonzero commuting derivation. It is also easy to show that if $D: R \rightarrow R$ is a commuting derivation on a semiprime ring $R$, then $D$ maps $R$ into $Z(R)$ (see, for example, the end of the proof of Theorem 2.1 in [25]). Furthermore, Brešar [7] proved that every additive commuting mapping of a prime ring $R$ is of the form $x \mapsto \lambda x+\zeta(x)$, where $\lambda$ is an element of the extended centroid $C$ and $\zeta: R \rightarrow C$ is an additive mapping. For results concerning commuting mappings, centralizing mappings and related problems we refer the reader to $[1,5-13,18,22-28]$ where further references can be found.

In [18] Vukman and the first named author generalized the result proved by Brešar and Hvala for prime rings [9].

Theorem 1 ([18, Theorem 2]). Let $R$ be a 2-torsion free semiprime ring. Suppose that an additive mapping $f: R \rightarrow R$ satisfies the relation

$$
\left[f(x), x^{2}\right]=0
$$

for all $x \in R$. Then $f$ is commuting on $R$.

This result motivated us to prove our main theorem.

Main Theorem. Let $n>1$ be a fixed integer and $R$ a $n$ !-torsion free semiprime ring. Suppose that an additive mapping $f: R \rightarrow R$ satisfies the relation

$$
\left[f(x), x^{n}\right]=0
$$

for all $x \in R$. Then $f$ is commuting on $R$.

Let us point out that the above theorem might be of some interest from the functional analysis point of view as well since $C^{*}$-algebras (moreover, semisimple Banach algebras) are semiprime.

\section{Proof of the main theorem}

Let $n>1$ be a fixed integer. Before proving our main theorem, let us fix some notation and write two results (Lemma 1 and Proposition 1) which we will need in the following. Let $m>1$ be an integer and $\mathbb{F}$ an arbitrary field. Then $M_{m}(\mathbb{F})$ denotes the algebra of all $m \times m$ matrices over the field $\mathbb{F}$. Recall that $Z\left(M_{m}(\mathbb{F})\right)=\mathbb{F} I$, where $I \in M_{m}(\mathbb{F})$ is the identity matrix. By $E_{i j} \in M_{m}(\mathbb{F})$, $1 \leq i, j \leq m$, we will denote the matrix with $(i, j)$-entry equal to one and all the others equal to zero.

Lemma 1. Let $R=M_{m}(\mathbb{F}), m>1$, and $A \in R$. Suppose that

$$
\left[A, X^{n}\right]=0
$$

for all $X \in R$. Then $A \in \mathbb{F} I$.

Proof. Let $P$ be an idempotent matrix in $M_{m}(\mathbb{F})$. Setting $X=P$ in (2) and multiplying left side by $(I-P)$, we see that $(I-P) A P=0$ for any idempotent matrix $P$. Thus, $A$ is a diagonal matrix. Note that $U A U^{-1}$ must 
be diagonal for each invertible element $U \in M_{m}(\mathbb{F})$, since $\left[U A U^{-1}, X^{n}\right]=0$ for all $X \in M_{m}(\mathbb{F})$. Write $A=\sum_{i=1}^{m} \alpha_{i} E_{i i}$, where $\alpha_{i} \in \mathbb{F}$. Then, for each $j>1$ the $(1, j)$-entry of $\left(I+E_{1 j}\right) A\left(I+E_{1 j}\right)^{-1}$ equals 0 . That is, $\alpha_{j}=\alpha_{1}$ for $j>1$. Hence, $A \in \mathbb{F} I$, as desired.

Proposition 1. Let $R$ be a non-commutative prime ring and $a \in R$ such that

$$
\left[a, x^{n}\right]=0
$$

for all $x \in R$. Then $a \in Z(R)$.

Proof. Suppose on the contrary that $a \notin Z(R)$. Then

$$
f(X)=\left[a, X^{n}\right]
$$

is a nontrivial generalized polynomial identity (in the following referred as GPI) for $R$. Using [14], $f(X)$ is also a GPI for $Q$. Denote by $F$ either the algebraic closure of $C$ or $C$ itself according to the cases when $C$ is either infinite or finite dimensional, respectively. Then, using a standard argument (e.g., see [19, Proposition]), $f(X)$ is also a GPI for $Q \oplus_{C} F$. Since $Q \oplus_{C} F$ is a centrally closed prime $F$-algebra [15, Theorem 2.5 and Theorem 3.5], by replacing $R$ and $C$ with $Q \oplus_{C} F$ and $F$, respectively, we may assume that $R$ is centrally closed and $C$ is either finite dimensional or algebraically closed. In a view of Martindale's theorem [20], $R$ is a primitive ring having a non-zero socle with $C$ as its associated division ring.

Since $a \notin C$, we have $[a, x] \neq 0$ for some $x \in \operatorname{soc}(R)$. By Litoff's theorem [16], there exists an idempotent $e \in \operatorname{soc}(R)$ such that $x, a x, x a \in e R e$. Note that $e f(e X e) e$ is a GPI for $R$. Thus, $\left[(e a e), X^{n}\right]$ is a GPI for $e R e$. Since $e R e \cong M_{m}(C)$ for some $m \geq 1$, eae is central in $e R e$ by Lemma 1 . It follows that there exists $c \in C$ such that $c e=e a e$. Hence, $c x=e a e x=e a x=a x$. Similarly, $x c=x e a e=x a e=e x a e=x a$. So $[a, x]=0$, a contradiction. Therefore, $a \in Z(R)$, as desired.

Remark. Let us point out that in Proposition 1 we have no restriction on the characteristic of a non-commutative ring $R$. But if $R$ is $2 n$ !-torsion free, then the above proposition is a direct consequence of Theorem 2.1 in [25] (see also Theorem 3 in [17] for the generalization). Namely, if we define an inner derivation $D: R \rightarrow R$ by $D(x)=[a, x]$, then $D\left(x^{n}\right)=\left[a, x^{n}\right]$. Therefore, if $\left[a, x^{n}\right]=0$, then $D\left(x^{n}\right) x+x D\left(x^{n}\right)=0$ for all $x \in R$ and, by [25, Theorem 2.1], $D(x)=[a, x]=0$ for all $x \in R$. Thus, $a \in Z(R)$.

Now we are ready to prove our main theorem. In the proof we will use some ideas similar to those used in [28].

Proof of Main Theorem. By semiprimeness of $R$, there exists a family of prime ideals $\left\{P_{\alpha}: \alpha \in I\right\}$ such that $\cap_{\alpha \in I} P_{\alpha}=\{0\}$. Without loss of generality, we may assume that prime rings $R / P_{\alpha}, \alpha \in I$, are 2-torsion free (see [2, p. 459]).

Now, let us fix an arbitrary $\alpha \in I$. It is sufficient to show that $[f(x), x] \in P_{\alpha}$ for all $x \in R$. Denote by $C$ the extended centroid of a prime ring $R / P_{\alpha}$ and 
by $A$ the central closure of $R / P_{\alpha}$. One can consider $A$ as a vector space over the field $C$ which can be regarded as a subspace of $A$. Thus, there exists a subspace $B$ of $A$ such that $A=B+C$. Let $\pi$ be the canonical projection of $A$ onto $B$. For $x \in R$ we shall write $\bar{x}$ for the coset $x+P_{\alpha} \in R / P_{\alpha}$. Replacing $x$ by $x+p$ in (1) we obtain

$$
\left[f(p), x^{n}\right] \in P_{\alpha}
$$

for all $x \in R$ and $p \in P_{\alpha}$. Therefore, $\left[\overline{f(p)}, \bar{x}^{n}\right]=0$ for all $x \in R$. Using Proposition 1, it follows that $\overline{f(p)}$ lies in the center of $R / P_{\alpha}$, which means that $[\overline{f(p)}, \bar{x}]=0$ for all $x \in R, p \in P_{\alpha}$. In particular, we have $\pi \overline{f(p)}=0$. This yields that the mapping $\bar{f}: R / P_{\alpha} \rightarrow A, \bar{f}(\bar{x})=\pi \overline{f(x)}$, is well defined. It is easy to verify that $\bar{f}$ is additive and satisfies $\left[\bar{f}(\bar{x}), \bar{x}^{n}\right]=0$ for all $x \in R$. Using [3, Theorem 1.1] it follows that $[\bar{f}(\bar{x}), \bar{x}]=0$ which in turn implies $[f(x), x] \in P_{\alpha}$. The proof is completed.

In [8], Brešar proved that there are no nonzero skew-commuting additive mappings on a 2-torsion free semiprime $\operatorname{ring} R$. In other words, if $R$ is a 2torsion free semiprime ring and $f: R \rightarrow R$ an additive mapping such that $f(x) x+x f(x)=0$ for all $x \in R$, then $f=0$. Motivated by this result, we conclude our paper with the following conjecture.

Conjecture. Let $n \geq 1$ be some fixed integer and let $R$ be a semiprime ring with suitable torsion restrictions. Suppose that an additive mapping $f: R \rightarrow R$ satisfies the relation

for all $x \in R$. Then $f=0$.

$$
f(x) x^{n}+x^{n} f(x)=0
$$

In the case $n=1$, the above conjecture has been proved by Brešar in [8].

Acknowledgement. The authors would like to thank the referee for his/her useful comments.

\section{References}

[1] M. Ashraf and J. Vukman, On derivations and commutativity in semi-prime rings, Aligarh Bull. Math. 18 (1999), 29-38.

[2] W. E. Baxter and W. S. Martindale III, Jordan homomorphisms of semiprime rings, J. Algebra 56 (1979), no. 2, 457-471.

[3] K. I. Beidar, Y. Fong, P.-H. Lee, and T.-L. Wong, On additive maps of prime rings satisfying the engel condition, Comm. Algebra 25 (1997), no. 12, 3889-3902.

[4] K. I. Beidar, W. S. Martindale III, and A. V. Mikhalev, Rings with generalized identities, Marcel Dekker, Inc., New York, 1996.

[5] M. Brešar, On a generalization of the notion of centralizing mappings, Proc. Amer. Math. Soc. 114 (1992), no. 3, 641-649.

[6] _ Centralizing mappings and derivations in prime rings, J. Algebra 156 (1993), no. $2,385-394$.

7] _ Commuting traces of biadditive mappings, commutativity preserving mappings and Lie mappings, Trans. Amer. Math. Soc. 335 (1993), no. 2, 525-546.

[8] _ On skew-commuting mappings of rings, Bull. Austral. Math. Soc. 47 (1993), no. 2, 291-296. 
[9] M. Brešar and B. Hvala, On additive maps of prime rings, Bull. Austral. Math. Soc. 51 (1995), no. 3, 377-381.

[10] $\_$, On additive maps of prime rings. II, Publ. Math. Debrecen 54 (1999), no. 1-2, 39-54.

[11] M. Brešar and J. Vukman, On some additive mappings in rings with involution, Aequationes Math. 38 (1989), no. 2-3, 178-185.

[12] _ On left derivations and related mappings, Proc. Amer. Math. Soc. 110 (1990), no. $1,7-16$

[13] — Derivations of noncommutative Banach algebras, Arch. Math. (Basel) 59 (1992), no. 4, 363-370.

[14] C. L. Chuang, GPIs having coefficients in Utumi quotient rings, Proc. Amer. Math. Soc. 103 (1988), no. 3, 723-728.

[15] J. S. Erickson, W. S. Martindale III, and J. M. Osborn, Prime nonassociative algebras, Pacific J. Math. 60 (1975), no. 1, 49-63.

[16] C. Faith and Y. Utumi, On a new proof of Litoff's theorem, Acta Math. Acad. Sci. Hungar 14 (1963), 369-371.

[17] A. Fošner, M. Fošner, and J. Vukman, An identity with derivations on rings and Banach algebras, Demonstratio Math. 41 (2008), no. 3, 525-530.

[18] A. Fošner and J. Vukman, Some results concerning additive mappings and derivations on semiprime rings, Publ. Math. Debrecen 78 (2011), no. 3-4, 575-581.

[19] P.-H. Lee and T.-L. Wong, Derivations cocentralizing Lie ideals, Bull. Inst. Math. Acad. Sinica 23 (1995), no. 1, 1-5.

[20] W. S. Martindale III, Prime rings satisfying a generalized polynomial identity, J. Algebra 12 (1969), 576-584.

[21] E. C. Posner, Derivations in prime rings, Proc. Amer. Math. Soc. 8 (1957), 1093-1100.

[22] J. Vukman, Commuting and centralizing mappings in prime rings, Proc. Amer. Math. Soc. 109 (1990), no. 1, 47-52.

[23] - On derivations in prime rings and Banach algebras, Proc. Amer. Math. Soc. 116 (1992), no. 4, 877-884.

[24] _ Derivations on semiprime rings, Bull. Austral. Math. Soc. 53 (1996), no. 3, $353-359$.

[25] - Identities with derivations on rings and Banach algebras, Glas. Mat. Ser. III 40(60) (2005), no. 2, 189-199.

[26] - On $\alpha$-derivations of prime and semiprime rings, Demonstratio Math. 38 (2005), no. $2,811-817$.

[27] - On left Jordan derivations of rings and Banach algebras, Aequationes Math. 75 (2008), no. 3, 260-266.

[28] J. Vukman and I. Kosi-Ulbl, On some equations related to derivations in rings, Int. J. Math. Math. Sci. 2005 (2005), no. 17, 2703-2710.

AJDA FOŠNER

FACUlTy of Management

University OF Primorska

CAnkarjeva 5, SI-6104 Koper, Slovenia

E-mail address: ajda.fosner@fm-kp.si

NADEEM UR REHMAN

Department of Mathematics

Aligarh Muslim University

Aligarh-202002, India

E-mail address: rehman100@gmail.com 